\title{
Experimental infection of inbred guinea pigs with Treponema pallidum: development of lesions and formation of antibodies
}

\author{
CHARLES S PAVIA AND CAROL J NIEDERBUHL \\ From the Trudeau Institute, Saranac Lake, New York, USA
}

SUMMARY Inbred strain 2 and strain 13 guinea pigs were infected intradermally in the hind legs with different numbers of the virulent Nichols strain of Treponema pallidum. About $91 \%$ of the animals developed clearly visible lesions after being injected with $5 \times 10^{6}$ to $10 \times 10^{6}$ treponemes. $T$ pallidum organisms could be isolated from skin lesions at various stages after infection. Infected animals were monitored for the production of specific treponemal and nonspecific cardiolipin antibodies by the fluorescent treponemal antibody (FTA) and microhaemagglutination (MHA-TP) tests and the rapid plasma reagin (RPR) card test. Low levels of specific antibodies could be detected by both FTA and MHA-TP tests from three to four weeks after infection. Maximum titres of treponemal antibody generally occurred after week 6 and persisted for several more months. These peak titres ranged from 1/40 to 1/80 in the FTA test and $1 / 160$ to $1 / 320$ in the MHA-TP test. During the same period infected guinea pigs, unlike rabbits with syphilis, did not produce detectable quantities of antibodies against cardiolipin.

\section{Introduction}

Although much clinical and experimental information on treponemal infection has accumulated and been reviewed, ${ }^{12}$ it is still not clear what roles humoral and cell mediated immunity have in infection with $T$ pallidum. This is partly because pure $T$ pallidum organisms devoid of host tissues are not readily cultivable in the quantities needed for detailed biological and immunological studies. Another important limiting factor has been the difficulty of developing suitable inbred animal models necessary to evaluate the immune mechanisms occurring during syphilis. Although considerable success has been achieved towards elucidating the nature of resistance to syphilis using inbred hamsters infected with $T$ pallidum subsp endemicum (formerly $T$ pallidum Bosnia A), the causative agent of non-venereal syphilis, ${ }^{34}$ these studies have not been confirmed experimentally using strains of $T$ pallidum that cause venereal syphilis.

Because of their well defined immunogenetics and inexpensive maintenance, mice would seem to be ideal for studying pathogenic mechanisms and

Address for reprints: Dr C S Pavia, Trudeau Institute Incorporated, PO Box 59, Saranac Lake, NY 12983, USA

Accepted for publication 6 July 1984 immune responses unique to syphilis. Except for one unconfirmed report, ${ }^{5}$ however, infection of mice with $T$ pallidum is subclinical because clearly visible darkfield positive lesions do not develop, ${ }^{67}$ which restricts their suitability for investigations with $T$ pallidum. In view of the paucity of relevant rodent models, we decided to evaluate inbred strains 2 and 13 guinea pigs by examining their response to infection with syphilis. Studies by other workers have already established that outbred guinea pigs are capable of developing symptomatic infection after exposure to $T$ pallidum organisms and that certain components of both humoral and cellular immunity become expressed in these animals during experimental disease. ${ }^{18-11}$ The results reported here show that intradermal infection with $T$ pallidum of animals belonging to these two inbred guinea pig strains regularly produced primary lesions that were comparable with those seen in similarly infected rabbits. We also present evidence of the gradual development of a specific humoral immune response by guinea pigs infected with $T$ pallidum.

\section{Materials and methods}

ANIMALS

Strain 2 guinea pigs were bred under barrier sustained conditions at the animal breeding facility of this 
institute from animals originally obtained from the division of research services, National Institutes of Health, Bethesda, Maryland, United States. Strain 13 guinea pigs were purchased from ARI Breeding Laboratories, East Bridgewater, Maine, United States. Strain 2 (GPLA (guinea pig leucocyte antigen)B 1, S, Ia 2,4,5,6) and strain 13 (GPLA-B 1, S, Ia $1,3,5,6,7)$ guinea pigs have been inbred since 1906 . Reciprocal skin grafts between these two strains are usually rejected within 17 days. ${ }^{12}$ Both strains share the serologically detectable histocompatibility antigens determined by the GPLA loci (equivalent to murine H-2K/D gene products) but not several other antigens (Ia), which are determined by complex linked genes in three subregions of the I region. We used adult (three to 12 month old) animals weighing 400 to $800 \mathrm{~g}$ and giving negative reactions in the rapid plasma reagin (RPR) card test (Fisher Diagnostics, Orangeburg, New York, United States), the fluorescent treponemal antibody (FTA) assay, or the microhaemagglutination treponemal antibody (MHA-TP) test (Sera-Tek; Ames Division, Miles Laboratories, Elkhart, Indiana, United States). Outbred New Zealand white male rabbits giving negative reactions to the RPR or MHA-TP tests were supplied by the animal breeding facility of this institute. All animals were housed in an air filtered environment maintained at a mean (SD) temperature of $20(2)^{\circ} \mathrm{C}$.

\section{BACTERIA AND INFECTIONS}

The virulent Nichols strain of $T$ pallidum was generously provided by Dr James Folds (University of North Carolina, Chapel Hill, United States). A sample of the original inoculum was found to be free of viruses known to be pathogenic to rodents according to results of routine serological screening performed by the animal diagnosic testing service of Microbiological Associates, Walkersville, Maryland. Before infecting guinea pigs, hair was removed from the areas chosen for the injection sites with electric clippers and close shaving with a razor and soap solution. Guinea pigs were inoculated intradermally with various numbers of treponemes (see Results) at multiple sites on the hind legs. Pathogenic organisms for these injections had been obtained by extraction of infected rabbit testicular tissue as described previously, ${ }^{13}$ except that treponemes were harvested in basal reduced medium ${ }^{14}$ is that contained RPMI 1640 instead of minimal essential medium. The extraction procedure was carried out at room temperature in an atmosphere of $1.5 \%$ oxygen, $5 \%$ carbon dioxide, and $93.5 \%$ nitrogen. Motile organisms were counted by the method of Fieldsteel et al, ${ }^{14}$ after being stained with acridine orange and viewed under ultraviolet illumination. ${ }^{16}$ The size of developing lesions was monitored against time by measuring changes in the diameter of the infected skin sites with dial calipers. In some experiments control animals received an intradermal injection of heat killed (at $56^{\circ} \mathrm{C}$ for one hour) $T$ pallidum or an inoculum of testicular extract obtained after subjecting a suspension of freshly harvested treponemes to high speed centrifugation (at $12800 \times g$ for 15 minutes). This supernatant was frozen and thawed several times and, on microscopical examination, contained no visible treponemes or only small numbers (less than $10^{4} / \mathrm{ml}$ ) of non-motile organisms.

\section{DETECTING ANTIBODIES}

Whole blood was collected from guinea pigs before infection, at weekly or biweekly intervals up to three months after inoculation, and at monthly intervals thereafter. The ear veins of guinea pigs were punctured with a 23 gauge needle, and the blood was aspirated into $250 \mu \mathrm{l}$ heparinised tubes (American Scientific Products, McGaw Park, Illinois, United States). Plasma was separated from cells by centrifuging (at $12800 \times g$ for five minutes) and stored frozen at $-20^{\circ} \mathrm{C}$ until tested. Plasma was assayed for the presence of antibody to cardiolipin by the RPR card test ${ }^{17}$ and for specific antitreponemal antibodies by the FTA ${ }^{18}$ and MHA-TP tests. The MHA-TP test was performed according to the instructions supplied by the manufacturer. Selected plasma samples were tested with doubling amounts of absorbing diluent until an end point was reached.

Commercially prepared slides coated with $T$ pallidum (Treposlides, Beckman Instruments, Fullerton, California, United States) and a fluoresceinated antiguinea pig IgG (US Biochemical Corporation, Cleveland, Ohio, United States) were used in the FTA test. In preliminary experiments, plasma samples taken from guinea pigs before infection had been tested for reactivity in the FTA test by being mixed with phosphate buffered saline (PBS) $\mathrm{pH} 7 \cdot 2$ at a final dilution of $1 / 20$. As these initial samples had given negative reactions, PBS was used instead of absorbing diluent to test plasma samples taken after infection for antitreponemal antibody in the FTA test. For comparison, other experiments were designed whereby rabbits infected with $T$ pallidum were also monitored for the formation of specific antitreponemal antibodies and the non-specific antibody to cardiolipin.

DETECTING TPALLIDUM ORGANISMS IN INFECTED LESIONS

At selected intervals after intradermal infection of guinea pigs, 10-20 $\mu \mathrm{l}$ samples of fluid were removed from scrapings of obvious skin lesions. These were mixed with an equal amount of acridine orange 


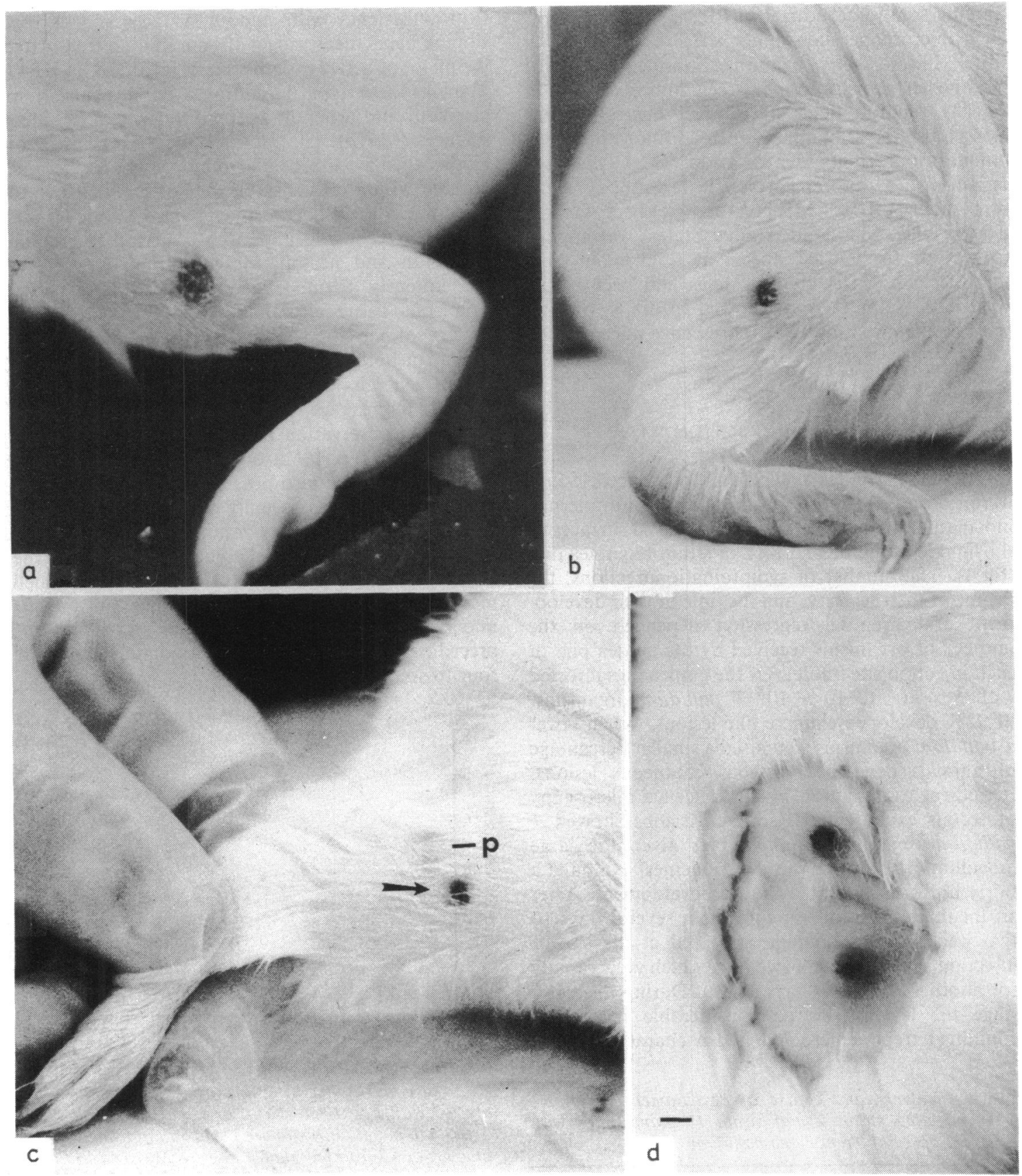

FIG 1 Representative examples of cutaneous lesions in (a) strain 13 guinea pig 11 days after infection with $5 \times 10^{6}$ $T$ pallidum in the left leg; (b) strain 2 guinea pig 18 days after infection with $10 \times 10^{6} T$ pallidum in the right leg; (c) strain 2 guinea pig with secondary lesion (arrow) developing adjacent to healed primary lesion (-p) eight weeks after a primary infection in the right leg; and (d) cutaneous lesions occurring on the shaven back of a New' Zealand white rabbit two to three weeks after infection with $10 \times 10^{6} \mathrm{~T}$ pallidum (har $=10 \mathrm{~mm}$ ). 
staining solution $(50 \mathrm{mg} / \mathrm{l}$ in PBS) and were placed on a microscope slide under a $22 \times 22 \mathrm{~mm}$ cover slip. Slides were examined immediately for treponemes showing bright green fluorescence after viewing random fields of samples microscopically using ultraviolet illumination. In addition, for direct immunofluorescent identification of $T$ pallidum organisms, a fluorescein labelled antibody to $T$ pallidum (BBL Microbiological Systems, Cockeysville, Maryland, United States) was applied to acetone fixed dried smears of fluid taken from these lesions. After treated slides had been rinsed with PBS, samples were allowed to dry in air and were then viewed under a fluorescence microscope.

\section{Results}

\section{CLINICAL COURSE OF INFECTION WITH $T$ PALLIDUM}

In our initial studies experimentally induced lesions were observed most readily after intradermal inoculation of $T$ pallidum into the shaved surfaces of the hind legs of either strain 2 or strain 13 guinea pigs (fig 1). The number of symptomatic infections, the degree of their severity, and the time of their development and eventual regression depended on the number of organisms received by the guinea pigs at each injection site (table). Of the guinea pigs infected with $5 \times 10^{6}$ to $10 \times 10^{6} T$ pallidum organisms, 91-92\% developed chancre like lesions. When fewer $T$ pallidum organisms were used a smaller percentage of animals developed obvious cutaneous lesions. Periodical examination by fluorescence microscopy of serous exudate from selected lesions showed $T$ pallidum organisms. Guinea pigs given the large inoculum $\left(5 \times 10^{6}\right.$ or more treponemes) exhibited a fairly uniform pattern of lesion development. After an incubation period ranging from several days to two weeks a small papule appeared at the site of injection as a prominent swelling, which grew rapidly for another five to 10 days (fig 2). During this early stage the lesions showed considerable reddening, contained treponemes, and had a characteristically

TABLE Incidence and course of development of lesions after injecting strain 2 and strain 13 guinea pigs with different doses of Treponema pallidum

\begin{tabular}{llll}
\hline $\begin{array}{l}\text { No of } \\
\text { organisms } \\
\text { injected }\end{array}$ & $\begin{array}{l}\text { No of } \\
\text { animals } \\
\text { infected }\end{array}$ & $\begin{array}{l}\text { No (\%) of } \\
\text { animals } \\
\text { with lesions }\end{array}$ & $\begin{array}{l}\text { Mean day } \\
\text { of first } \\
\text { appearance } \\
\text { of lesions }\end{array}$ \\
\hline $10 \times 10^{6}$ & 22 & $20(91)$ & 9 \\
$5 \times 10^{6}$ & 11 & $11(92)$ & 9 \\
$1-2 \times 10^{6}$ & 8 & $8(80)$ & 12 \\
$0.1 \times 10^{6}$ & 4 & $4(57)$ & 26 \\
\hline
\end{tabular}

*All animals were injected intradermally in the hind legs. firm consistency with smooth surfaces and narrow sloping bases measuring 4-10 $\mathrm{mm}$ in diameter. After the phase of active progression of infection, at weeks 2 to 4 , these typical indurated papules began to discolour and generally became wider with irregular surfaces (fig $1 \mathrm{~b}$ ). Ulceration and severe necrosis of lesions then occurred, and they sometimes became covered with a crusted erosion. For the next two to three weeks these haemorrhagic and necrotic lesions began to recede and underwent a period of gradual regression and healing. Scars with scaly surfaces subsequently formed, after which the affected cutaneous tissue virtually completely healed. The pattern of development of lesions appeared to be similar in both strains of guinea pig, although skin lesions generally persisted longer (up to week 10) in strain 13 guinea pigs. It should be noted that during a six month observation period lesions recurred in a small percentage $(10 \%)$ of infected animals. These lesions, which can also possibly be classified as late developing chancres, usually appeared two to four weeks after the primary lesions had receded and healed, and they developed adjacent to the site of the former primary lesions (fig 1c). They also contained motile treponemes. These secondary eruptions underwent a clinical course similar to that of the preceding primary lesion and ultimately regressed four to six weeks later.

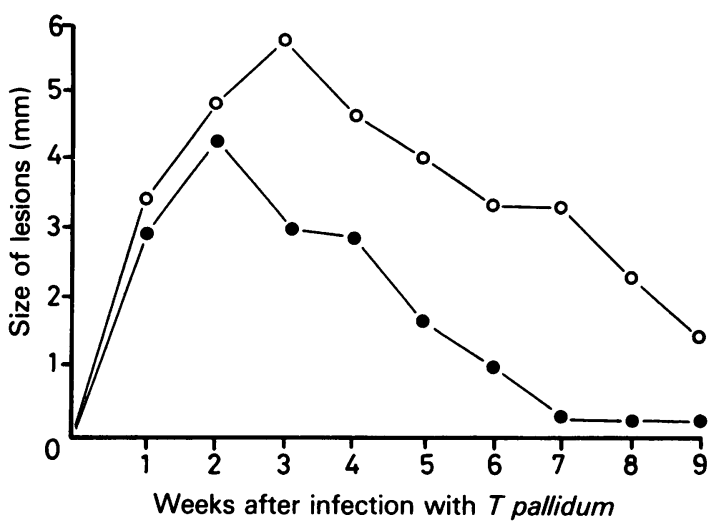

FIG 2 Size and course of lesions occurring in guinea pigs infected with $1 \times 10^{6}\left(-\mathrm{O}^{-}\right)$or $5 \times 10^{6} 1010 \times 10^{6}$ (-) $T$ pallidum. Data are mean values using the same group of infected guinea pigs as described in the table. Standard deviation for each time point was $\leqslant 15 \%$.

In some experiments guinea pigs used as noninfectious controls were injected intradermally with either $5 \times 10^{6}$ heat killed T pallidum or $0.1 \mathrm{ml}$ of an extract of infected rabbit testes that lacked motile treponemes. For a transient period lasting three to six days after inoculation, most animals reacted to these 
injections by exhibiting only slight erythema of the skin surface, which disappeared shortly thereafter. Rabbits infected intradermally with virulent $T$ pallidum under conditions identical to those used for strain 2 and 13 guinea pigs developed lesions (fig 1d) that resembled those occurring in guinea pigs with syphilis but were slightly larger, measuring $7-15 \mathrm{~mm}$ in diameter.

\section{HUMORAL RESPONSES}

On the basis of previous results, groups of both strain 2 and 13 guinea pigs were injected intradermally at multiple (two to four) sites with either $5 \times 10^{6}$ or $10 \times 10^{6} T$ pallidum organisms. The animals were bled weekly or biweekly, and their plasma was examined for the presence of specific antibodies directed against $T$ pallidum and for nonspecific cardiolipin antibodies. The formation of specific treponemal antibodies was monitored by the FTA technique and by the MHA-TP procedure. All plasma samples were initially diluted to $1 / 20$ in the FTA test and 1/80 in the MHA-TP assay.

As early as three to four weeks after infection, measurable amounts of specific antitreponemal antibodies were detected in both the FTA and MHA-TP tests (fig 3) in all plasma samples from infected guinea pigs. Before then antibodies had been found in the plasma of only a small percentage $(11 \%)$ of guinea pigs with syphilis. This level of antibody production was followed by a gradual rise in antibody titre for the next four weeks until maximum concentrations were reached, usually six to eight weeks after infection. These peak levels persisted over the ensuing months. The MHA-TP procedure showed that rabbits similarly infected with syphilis developed antibody more rapidly (fig 3 ). Ten days after infection, serum samples from rabbits with syphilis attained mean titres as high as $1 / 1280$.

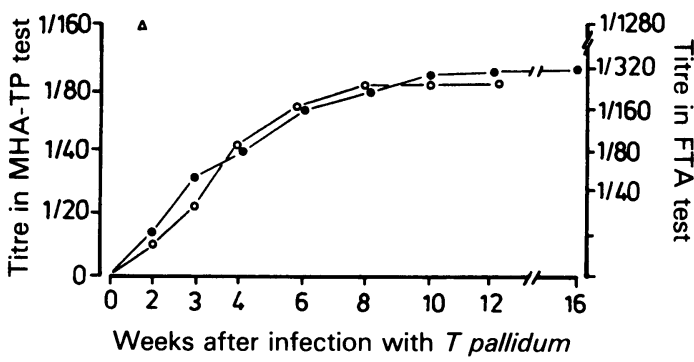

FIG 3 Antitreponemal antibody response of 18 guinea pigs infected with $T$ pallidum using the FTA (- $\bigcirc-)$ and $\left.M H A-T P(-)_{-1}\right)$ tests and of five rabbits infected with $T$ pallidum using the MHA-TP test $(\Delta)$. Each point represents the mean of the highest dilution showing at least $l+$ reactivity.
In noticeable contrast, antibody to cardiolipin measured by the RPR card test could not be detected in the plasma of guinea pigs infected with $T$ pallidum during an observation period of 15 weeks after infection (fig 4). In further experiments (data not shown) plasma samples of animals with syphilis examined after this period (at 20 and 24 weeks) were also found to be negative for reagin, and cardiolipin antibodies did not develop in guinea pigs that had received a challenge inoculum of $T$ pallidum organisms. These findings differed sharply from the response pattern of rabbits undergoing similar cutaneous infections, in which antibodies to cardiolipin started to develop two to three weeks after infection (fig 4), a relatively high titre of reagin was maintained for the following four to five weeks, and this declined slightly for the remaining seven weeks of the experiment.

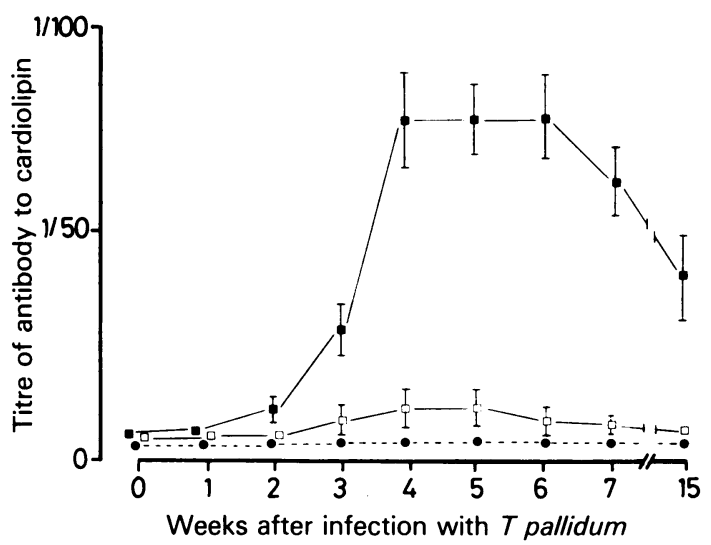

FIG 4 Development of antibody reacting with cardiolipin in the RPR card test in 18 guinea pigs infected with $T$ pallidum (-O-), five rabbits infected with $T$ pallidum (- - ), and five rabbits injected with heat killed treponemes $(-\square-)$. Each point represents the mean of the highest dilution showing reactivity.

\section{Discussion}

Infection of the guinea pig with $T$ pallidum has yet to be well established as an experimental model of venereal syphilis in man, despite early 89 and recent ${ }^{1011}$ reports that attest to the susceptibility of the cavy to treponemal infection. This appears to be because guinea pigs are thought to be less susceptible than rabbits to infection with syphilis ${ }^{12}{ }^{11}$ or because the tissue lesions are atypical compared with those of syphilis in man, ${ }^{2}$ the natural host for $T$ pallidum. Studies conducted more than three decades ago reported difficulty in producing clinically apparent disease in guinea pigs with syphilis, ${ }^{1} 1920$ although 
various tissues including draining lymph nodes contained infectious organisms. In this respect we have recovered viable treponemes from the draining inguinal lymph nodes of strain 2 guinea pigs during the early stages of infection with syphilis (Pavia, unpublished observation). In some experiments only a relatively small proportion of infected guinea pigs developed dark field positive lesions. ${ }^{1}$ In many of these reports, however, it was difficult to assess the infective dose of treponemes that was critical in the induction and pathogenesis of symptomatic infection. ${ }^{120}$

A recent report showed that nearly $80 \%$ of outbred Hartley guinea pigs developed lesions when injected in the pubic region with $0.5 \times 10^{6}$ or more treponemes. ${ }^{11}$ This confirmed reports by Wicher et al that $77 \%$ of their guinea pigs became infected after inoculation with $20 \times 10^{6}$ treponemes. ${ }^{10} \mathrm{We}$ are not aware of any previous investigations describing $T$ pallidum infection in inbred or genetically defined strains of guinea pigs. Our findings with strain 2 and 13 guinea pigs agreed with the results obtained in more recent reports ${ }^{10}{ }^{11}$ rather than with some of the earlier, frequently unsuccessful, attempts to infect guinea pigs with $T$ pallidum. ${ }^{19}{ }^{20} \mathrm{We}$ found that after being injected with $5 \times 10^{6}$ to $10 \times 10^{6} T$ pallidum organisms, more than $90 \%$ of inbred guinea pigs developed skin lesions whose pattern in some instances exhibited considerable chronicity. The reasons for discrepancies among these various studies are not entirely clear. Continued refinement of the extraction and harvesting of treponemes from infected rabbit testes in solutions or media and under atmospheric conditions that promote survival of virulent organisms ${ }^{14} 15$ undoubtedly influences the overall quality of treponemal preparations. Current studies are likely to have a distinct advantage over earlier ones because our increased knowledge about the biological properties and requirements for growth of $T$ pallidum may have resulted in improvements over past preparative procedures and better preservation of the overall integrity of treponemal organisms. The variations in development of lesions in guinea pigs that occurred in these several studies may therefore reflect differences in numbers or virulence of viable spirochaetes recovered during the process of harvesting from orchitic rabbit tissue.

We have observed that skin lesions containing treponemes in guinea pigs with syphilis resembled those occurring in similarly infected rabbits (fig 1), although the severity, duration, and overall size of individual lesions sometimes varied. Such chancre like lesions have previously been shown to cause considerable histopathological changes within the various layers of guinea pig skin. ${ }^{11}$ Interestingly, in some of our animals infection with syphilis took a more chronic course, with lesions persisting for several weeks after the initial appearance of the primary lesion. Nevertheless, there were some notable differences in the pattern of expression of humoral immunity between guinea pigs and rabbits during their response to infection with syphilis. Unlike experimentally infected rabbits or patients with syphilis, guinea pigs infected with $T$ pallidum did not seem to produce antibody against cardiolipin (fig 4). ${ }^{1011}$ This finding was similar to that in other rodents, which failed to generate an anticardiolipin humoral response during treponemal infection. ${ }^{21}$

There is still no satisfactory explanation for this different pattern, although it suggests that the guinea pig is a poor responder to lipid components present either on treponemes or components derived from the host that may become altered during syphilitic infection. In addition, guinea pigs with syphilis appear to mount a less vigorous antibody response against specific treponemal antigens. Usually at least three to four weeks elapse before guinea pigs infected with $T$ pallidum begin to synthesise concentrations of antibodies detectable by both the FTA and MHA-TP tests (fig 3), ${ }^{11}$ whereas rabbits begin to produce comparatively high concentrations of antitreponemal antibodies within 10 days after infection (fig 3). ${ }^{22}$ Nevertheless, guinea pigs with syphilis do retain substantial antibody reactivity against treponemal antigens for at least five months after infection, and the kinetics of this humoral immune response are similar to those previously reported for the outbred Hartley strain of guinea pigs infected with compar-able numbers of $T$ pallidum organisms. ${ }^{1011}$

The adaptation of inbred guinea pigs for studying the mechanisms of immunity to infection with $T$ pallidum has several possible advantages over other experimental systems. Firstly, the elucidation of protective mechanisms that operate during syphilis has been hampered by lack of suitable animal models. As in studies in man, most current information is based primarily on experimental infection of the rabbit. Yet there are certain inconsistencies in data related mostly to cellular immune aspects, ${ }^{2}$ which can be partly attributed to using mixed patient populations and outbred animals. Although the rabbit has long been considered to be and used as the animal model of choice, there still remain several important shortcomings with this experimental system. Compared with most readily available rodents, rabbits are very expensive to obtain and house, and their immune system is not as well defined. Strain 2 and strain 13 guinea pigs can be obtained easily, are not as expensive as rabbits to purchase and maintain, and their immunogenetics are fairly well defined. Furthermore, among small laboratory animals the guinea pig most closely resembles man in terms of 
hormonal balance, reproductive and skin physiology, immune responses, and certain nutritional requirements. ${ }^{13}$ The major advantage of using inbred guinea pigs is the possibility of performing cellular adoptive transfer experiments (which cannot be done effectively with outbred rabbits) to assess the relative importance of humoral and cellular expressions of immunity to syphilis. Secondly, only limited success has been achieved when other inbred rodents have been evaluated for their usefulness in syphilis research. Klein et al showed that some strains of mice were more susceptible than others to symptomatic infection with $T$ pallidum, ${ }^{5}$ which led to optimistic forecasts of rapid development of the mouse for studying immune phenomena associated with syphilis. The future outlook for a workable murine model, however, has been seriously questioned by a more recent and extensive investigation that was unable to detect lesions in over $\mathbf{4 0 0}$ mice infected with $T$ pallidum, including mice with known immune defects. ${ }^{7}$ Apart from that series of experiments in mice, the only set of extensive investigations using inbred animals to study host defence mechanisms against syphilis was that of Schell et al using hamsters. ${ }^{3423}$ Their findings clearly showed the contribution that both humoral and cell mediated immunity make in protecting the host against syphilitic infection.

Those experiments, however, were restricted primarily to the use of the Bosnia A strain of $T$ pallidum (agent of endemic syphilis), whereas most of the current body of information has been derived from studies of immunity after infection with the Nichols strain of $T$ pallidum (agent of venereal syphilis) adapted in the rabbit. In fact, this organism rarely causes clinical disease in experimentally infected hamsters, ${ }^{23}$ which poses some limitations in relating the interesting findings of Schell $e t$ al to the preceding results other workers obtained with $T$ pallidum Nichols strain or other venereal strains of this organism. In view of these facts and the ease with which symptomatic treponemal infection can be induced in guinea pigs, we think that this experimental animal model has great potential for further research into syphilis. In this regard it should provide a better understanding of some of the basic underlying immune mechanisms responsible for acquired protection against syphilitic infection, which contribute to the destruction of the pathogenic spirochete or help explain the delay in effective antitreponemal immune responses occurring in the diseased host.

This work was supported by Public Health Service grant AI-19824 to CSP from the National Institute of Allergy and Infectious Diseases.

\section{References}

1. Turner TB, Hollander DH. Biology of the treponematoses. WHO Monograph Series 1957; 35: 1-277.

2. Schell RF, Musher DM, eds. Pathogenesis and immunology of treponemal infection. New York: Marcel Dekker, 1983.

3. Schell RF, Chan JK, LeFrock JL. Endemic syphilis: passive transfer of resistance with serum and cells in hamsters. $J$ Infect Dis 1979; 140: 378-83.

4. Schell RF, Chan JK, LeFrock JL, Bagasra O. Endemic syphilis: transfer of resistance to Treponema pallidum strain Bosnia A in hamsters with a cell suspension enriched in thymus-derived cells. J Infect Dis 1980; 141:909-13.

5. Klein J, Monjan AA, Hardy PH, Cole GA. Abrogation of genetically controlled resistance of mice to Treponema pallidum by irradiation. Nature 1980; 283:572-4.

6. Schell RF, Musher DM, Jacobson K, Schwethelm P. New evidence for the non-infectivity of Treponema pallidum for mice. British Journal of Venereal Diseases 1975;51:19-21.

7. Folds JD, Rauchbach AS, Shores E, Saunders JM. Evaluation of the inbred mouse as a model for experimental Treponema pallidum infection. Scand J Immunol 1983; 18:201-6.

8. Kolle W, Evers E. Experimentalle Studien uber Syphilis und Rekurrensspirochaetose. Dtsch Med Wochenschr 1926; 26: 1075-6.

9. Tani T, Kakishita M, Saito K. Beitrage zur Meerschweinchen syphilis. Zentralbl Bakteriol Mikrobiol Hyg 1930;117:73-9.

10. Wicher K, Wicher V, Wang MCC. Cellular and humoral immune response of guinea pig infected with Treponema pallidum. Int Arch Allergy Appl Immunol 1976;51:284-97.

11. Pierce CS, Wicher K, Nakeeb S. Experimental syphilis: guinea pig model. British Journal of Venereal Diseases 1983;59: $157-68$.

12. Wagner JE, Manning PJ, eds. Biology of the guinea pig. New York: Academic Press, 1976.

13. Pavia CS, Folds JD, Baseman JB. Selective in vitro response of thymus-derived lymphocytes from Treponema pallidum infected rabbits. Infect Immun 1977; 18:603-11.

14. Fieldsteel AH, Becker FA, Stout JG. Prolonged survival of Treponema pallidum (Nichols strain) in cell-free and tissue culture systems. Infect Immun 1977; 18: 173-82.

15. Norris SJ. In vitro cultivation of Treponema pallidum: independent confirmation. Infect Immun 1982;36:437-9.

16. Pavia CS, Diggs CL, Williams J. The use of metrizamide for isopyknic separation and enrichment of Plasmodium falciparum schizonts from continuous culture. Am J Trop Med Hyg 1983; 32:675-81.

17. March RW, Stiles GE. The reagin screen test: a new reagin card test for syphilis. Sex Transm Dis 1980; 7:66-70.

18. Hunter E, Deacon W, Meyer P. An improved FTA test for syphilis, the absorption procedure (FTA-ABS). Public Health Rep 1964;79:410-2.

19. Dold H, Worms W. Zur Frage des Verhaltens der Spirochaeta pallidum in Meerschweinchen. Dtsch Med Wochenschr 1928; 47: 1962-3.

20. Gastinel P, Vaisman A, Vaisman H. La dispersion treponemique dans la syphilis experimental du cobaye. Annales de Dermatologie et de Syphiligraphie 1955;82:481-3.

21. Levaditi C, Vaisman R, Schoen R, Manin Y. Tentative de transmission hereditaire de l'infection syphilitique inapparente chez la souris blanche. Annales de Institut Pasteur 1935;54: 584-626.

22. Pepose JS, Bishop NH, Feigenbaum S, Miller JN, Zeltzer PM. The humoral immune response in rabbits infected with Treponema pallidum: comparison of antibody levels measured by the staphylococcal protein A-IgG (SPA-TP) microassay with VDRL, FTA-ABS, and TPI antibody responses during the development of acquired resistance to challenge. Sex Transm Dis 1980; 7: 125-9.

23. Schell RF, LeFrock JL, Chan JK, Bagasra O. LSH hamster model of syphilitic infection. Infect Immun 1980;28:909-13. 